\title{
A clinical and bacteriological study of bacterial folliculitis
}

\author{
Lokesh S Jappa ${ }^{1, *}$, Sameer R Kutre ${ }^{2}$ \\ ${ }^{1}$ Associate Professor, Dept. of Dermatology, STD, Leprosy, NKP Salve Institute of Medical Sciences and Research Centre, \\ Nagpur, Maharashtra, India, ${ }^{2}$ Private Practitioner
}

*Corresponding Author:

Email: drlokeshsid@gmail.com

\begin{abstract}
Folliculitis constitutes a major component of pyoderma which is mostly caused by S. Aureus. However clinical and bacteriological studies are mostly lacking. This study was taken up to evaluate the proportion of various clinical types of bacterial folliculitis to decide regarding the most appropriate antibiotic of choice for folliculitis. A total of 100 untreated patients of folliculitis were enrolled in the study. Detailed history and clinical examination was done. Antibiotic sensitivity of pus was done when pus formation was evident in all cases of folliculitis. In present study, proportion of folliculitis was $5.61 \%$. Folliculitis was commonest in the age group of 21 to 30 years. Sex ratio of male to female was 2.5:1. Folliculitis was more commoner in low socioeconomic status (69\%). While it was most commonly seen in monsoon (49\%). Positive family history was seen in $33 \%$ patients. Superficial folliculitis was commonest in children. Chronic folliculitis was mostly seen in adult males. Staph. aureus was isolated in $89 \%$ patients (including mixed growth of S. aureus and beta haemolytic streptococci) and Staph. epidermidis in 9\% cases of folliculitis (including mixed growth of Staph. epidermidis and Beta haemolytic streptococci), Beta haemolytic streptococci was seen as mixed isolate in $7 \%$ cases. This study favours using cephalosporins as the first line of treatment in folliculitis on the basis of emergence of resistance strains. This study demonstrated the need of continuous monitoring of the changing pattern of antibiotic sensitivity.
\end{abstract}

Keywords: Folliculitis, Staphylococcus Aureus, Sensitivity and resistance.

\section{Introduction}

In dermatology practice, cutaneous infections form the major part of patients attending the out-patient department. Amongst infections, cutaneous bacterial infections are more common of which folliculitis affecting hair follicle is commonly seen as perifollicular pustule. Folliculitis is defined as inflammation of hair follicle. It is most commonly caused by staphylococcus aureus, rarely by staphylococcus epidermidis and other gram negative bacteria. ${ }^{1,2}$ Clinically bacterial folliculitis is classified into superficial and deep folliculitis. Superficial type is further classified into Bokhart's impetigo and chronic superficial folliculitis of legs. Deep type is classified as sycosis barbae, furunculosis and carbuncle. Other types of bacterial folliculitis are gram negative folliculitis and Propionibacterium acne folliculitis. Folliculitis can extend to spread infection in surrounding perifollicular dermis leading to furunculosis. Hence it is important to correctly diagnose and treat folliculitis. More importantly in early stages, presence of pus makes bacteriological analysis more easy.

Various factors affecting folliculitis includes physical factors, age, sex, occupation, presence of carriers, drugs, and associated systemic diseases including immunodeficiency syndromes. Indiscriminate use of antibiotics has lead to both topical as well as systemic drug resistance. ${ }^{1-7}$ Aim of the current study was to study incidence rate of folliculitis amongst patients visiting dermatology clinic and to study various etiological factors and to study sensitivity and resistance pattern of causative organism.

\section{Materials and Methods}

Study group included 100 patients of primary folliculitis attending outpatient department of tertiary care hospital and referral centre in south India for a period of one year. All patients of clinically diagnosed folliculitis attending skin OPD who were untreated for the disease were enrolled in the study. Patients of nonfollicular bacterial infections like impetigo, ecthyma, erysipelas, cellulitis or necrotizing fasciitis and patients who were treated earlier with topical or systemic antibacterials were excluded. Most of the folliculitis cases are superficial characterized by follicular pustule. However there is special entity called as chronic recurrent folliculitis of legs characterized by recurrent itchy follicular pustules over legs. This was included as separate entity in this study. Furunculosis is a type of bacterial folliculitis which ruptures to cause severe perifolliculitis that is more painful and may cause puspointing at the top of follicular nodule. Carbuncle is the most severe type of folliculitis where multiple furuncles coalesce to form multiple pus-discharging nodules and necrosis. This is uncommon and commonly seen in diabetes. Detailed history of duration of the disease, mode of onset, progression of lesions, personal hygiene, socioeconomic status, predisposing factors like atopy, diabetes, HIV/AIDS, anemia etc., history of similar disease amongst family members or in past etc. was taken. Thorough clinical examination was carried with regard to general condition of patients, morphology and distribution of lesion, lymph node status. Skin smear from pustule was done for Gram staining along with samples for pus culture and antibiotic sensitivity were taken wherever there was evident pus formation. 
Antibiotic sensitivity to common antibiotics was done. However, unfortunately, this was not uniform in every case.

Specimen for culture is collected with the help sterile swabs after cleansing sterile pustule with $70 \%$ alcohol, follicle was ruptured using a sterile needle and cotton swabs. It was transported in autoclaved plain tube. A few specimen were directly smeared and gram stained, examined under oil immersion for morphology characteristics and specimen are inoculated in blood agar and MacConkeys agar, incubated at 37 degree C. aerobically for $24 \mathrm{hr}$ and bacteria were indentified. S. aureus was identified as golden yellow circular measuring 2-4 convex, smooth colonies whereas S. epidermidis was identified as white colonies. Later on biochemical characteristics were studied like mannitol fermentations test, slide coagulase and tube coagulase test. Antibiotic sensitivity was done by disc diffusion technique.

\section{Results}

The total number of patients attending the OPD during the study period was 17623 . Among these, the number of primary bacterial infections including follicular and non-follicular pyoderma were 989 forming $5.61 \%$ of the total patients seen in dermatology OPD. We observed a total of 100 patients with primary bacterial folliculitis as mentioned in the inclusion criteria above. Amongst 100 patients, a total of $69 \%$ were from rural and $31 \%$ were from urban areas. Amongst 100 patients, 72 males and 28 were females. Age distribution of the patients of folliculitis is shown in Table 1. Bacterial folliculitis was commonest in the age group of 21-30 years in which 33 patients (33/100) were affected followed by the age group of 11-20 years (19/100). With regard to associated symptoms, itching was the commonest symptom in 6 patients of superficial folliculitis while pain was the commonest symptom in furunculosis. Lymphadenitis was in 6 cases of severe furunculosis. Amongst patients of bacterial folliculitis, $20 \%$ were labourers, $27 \%$ were students, $21 \%$ were agriculturists, $12 \%$ were housewives and rest of $20 \%$ belonged to categories other than above. Frequency of patients of bacterial folliculitis was more in monsoon season where 49 cases were seen while 30 cases were seen in summer and 21 cases were observed in winter and family history is present amongst 39 cases while there was no family history in rest of 61 cases.

The most common site of involvement of bacterial follculitis was legs $(47 / 100)$, followed by forearm $(17 / 100)$, and thigh (12/100). In rest of the patients i.e.24, bacterial folliculitis was seen at other areas. The least common site observed in these 24 cases was axilla where 3 patients had bacterial follilcitis (3/100).

Table 1: Age Distribution of patients with bacterial folliculitis

\begin{tabular}{|l|c|c|c|c|c|c|c|c|c|c|}
\hline Age groups (in yrs) & $\mathbf{1 - 1 0}$ & $\mathbf{1 1 - 2 0}$ & $\mathbf{2 1 - 3 0}$ & $\mathbf{3 1 - 4 0}$ & $\mathbf{4 1 - 5 0}$ & $\mathbf{5 1 - 6 0}$ & $\mathbf{6 1 - 7 0}$ & $\mathbf{7 1 - 8 0}$ & $\mathbf{8 0 - 9 0}$ & $\mathbf{9 0 - 1 0 0}$ \\
\hline Sup.Folliculitis & 6 & 6 & 10 & 4 & - & - & - & - & - & - \\
\hline Chronic folliculitis of legs & - & 1 & 1 & 1 & & & & & - & - \\
\hline Sycosis barbae & - & - & 3 & 3 & 1 & - & - & - & - & - \\
\hline Furunculosis & 4 & 12 & 18 & 9 & 4 & 4 & 3 & 2 & - & - \\
\hline Canbuncle & - & - & 1 & 1 & 2 & 2 & 1 & 1 & - & - \\
\hline Total & 10 & 19 & 33 & 18 & 7 & 6 & 4 & 3 & - & - \\
\hline
\end{tabular}

The commonest type of folliculitis was superficial folliculitis and Furunculosis mainly affecting in males

while chronic folliculitis of legs and Sycosis barbae was exclusively A noted in males as shown in Table 2.

Table 2: Types of folliculitis and sex distribution in study population

\begin{tabular}{|l|c|c|}
\hline \multicolumn{1}{|c|}{ Clinical types } & \multicolumn{2}{c|}{ Sex distribution } \\
\hline & Males & Females \\
\hline Folliculitis $(\mathrm{n}-26)$ & 17 & 9 \\
\hline Chronic Folliculitis of legs $(\mathrm{n}=3)$ & 3 & - \\
\hline Sycosis Barbae $(\mathrm{n}=7)$ & 7 & - \\
\hline Furunculosis $(\mathrm{n}=56)$ & 39 & 17 \\
\hline Carbuncle $(\mathrm{n}=8)$ & 6 & 2 \\
\hline Total 100 & 72 & 28 \\
\hline
\end{tabular}

The most common associated disease observed was anemia in 25 cases of which it was commonly associated with furunculosis (15/25) followed by superficial folliculitis $(9 / 25)$. History of atopy was seen in 21 patients $(21 \%)$ of which it was commonly associated with furunculosis (14/21) followed by superficial folliculitis (7/21). Diabetes was most commonly seen in 20 patients of folliculitis of which 11 had furunculosis (perifollicutis) and 8 had carbuncle (coalition of perifolliculitis). HIV/AIDS was seen in one patient each of superficial folliculitis, furunculosis and sycosis barbae. Details of associated conditions with folliculitis are given in Table 3. 
Table 3: Associated conditions in folliculitis

\begin{tabular}{|l|c|c|c|c|}
\hline \multicolumn{1}{|c|}{ Folliculitis } & Anaemia & Atopy & Diabetes & HIV \\
\hline Superficial folliculitis & 9 & 7 & 1 & 1 \\
\hline Chronic folliculitis of legs & - & - & - & - \\
\hline Sycosis barbae & - & - & - & 1 \\
\hline Furunculosis & 15 & 14 & 11 & 1 \\
\hline Carbuncle & 1 & - & 8 & - \\
\hline Total & 25 & 21 & 20 & 3 \\
\hline
\end{tabular}

Gram staining revealed Gram positive cocci in 73 patients while it was found to be negative in remaining of 27 patients. Pus culture sensitivity analysis revealed single organism Staph. Aureus (SA) in 83 patients and
Staph. Epidermidis (SE) in 8 patients and followed by mixed growth in 7 patients and no growth was seen in 2 patients (Table 4). Staphylococcus aureus was the most common organism isolated.

Table 4: Bacteriological analysis is given in table 3

\begin{tabular}{|l|c|c|c|c|c|c|c|c|c|c|}
\hline Folliculitis & SA & $\mathbf{\%}$ & SE & $\mathbf{\%}$ & SA+BHS & \% & SE+BHS & \% & No.organism & \% \\
\hline Sup.Folliculitis & 20 & 76.9 & 5 & 19.2 & 1 & 3.8 & - & - & - & - \\
\hline Chronic Folliculitis of legs & 3 & 100 & - & - & - & - & - & - & - & - \\
\hline Sycosis barbae & 7 & 100 & - & - & - & - & - & - & - & - \\
\hline Furunculosis & 46 & 81.1 & 3 & 5.3 & 4 & 7.1 & 1 & 1.7 & 2 & 3.5 \\
\hline Canbuncle & 7 & 87.5 & - & - & 1 & 12.5 & - & - & - & - \\
\hline Total & 83 & - & 8 & - & 6 & - & 1 & - & 2 & - \\
\hline
\end{tabular}

Sensitivity pattern of organisms to various antibiotics is given in Table 5. Accordingly, S. aureus was found to be resistant to ampicillin in about 76 cases when culture sensitivity was done in 83 patients in whom single organism staph. Aureus was detected.
Maximum sensitivity to $\mathrm{S}$. aureus was seen with netilimycin followed by ciprofloxacin and ceftriaxone. Maximum sensitivity to S. epidermidis was seen with netilimycin followed by ciprofloxacin and gentamycin.

Table 5: Sensitivity and resistance pattern of staphylococcus aureus, S. epidermidis and mixed isolates

\begin{tabular}{|l|c|c|c|c|c|c|c|c|c|}
\hline \multicolumn{1}{|c|}{ Antibiotics } & \multicolumn{3}{c|}{ S. Aureus } & \multicolumn{3}{c|}{ S.epidermidis } & \multicolumn{3}{c|}{ Mixed Isolates } \\
\hline Ampicillin & S & I & R & S & I & R & S & I & R \\
\hline Erythromycin & 5 & 2 & 76 & 2 & 2 & 4 & 1 & - & 6 \\
\hline Gentamycin & 27 & 17 & 35 & 3 & 3 & 1 & 3 & - & 4 \\
\hline Tetracycline & 60 & 3 & 15 & 7 & 1 & - & 6 & - & 1 \\
\hline Penicillin G & 26 & 1 & 42 & 3 & - & 4 & 1 & - & 6 \\
\hline Ciprofloxacin & - & - & 27 & - & - & 3 & - & - & - \\
\hline Netromycin & 72 & 3 & 6 & 8 & - & - & 7 & - & \\
& 76 & - & 4 & 8 & - & - & 7 & - & - \\
\hline Ceftriaxone & 69 & - & 5 & 4 & - & 1 & 6 & - & 1 \\
\hline Cefixime & 62 & - & 7 & 5 & - & 1 & & & \\
\hline Total & & & & & & & & & \\
\hline
\end{tabular}

\section{Discussion}

The current study was done in a tertiary care hospital and referral centre in south India. In the present study, the incidence of folliculitis is $5.61 \%$.Incidence of folliculitis among pyodermas quoted by other authors and in our study concurrence was $37.42 \%$ with study of

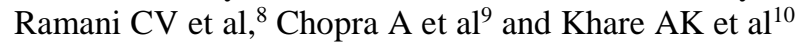
reported incidence of superficial folliculitis as $39.29 \%$ and $30.49 \%$. Findings coincide with present study having $26 \%$ of superficial folliculitis out of 100 patients. In present study incidence of recurrent folliculitis of leg was $3 \%$ which agrees with studies of
Bhat BK et al. ${ }^{11}$ The incidence in various parts of India is different. ${ }^{12}$

Chopra $\mathrm{A}$ et $\mathrm{al}^{9}$ have reported proportion of sycosis barbae $8.93 \%$ which correlates with the present study where the incidence was found to be $8 \%$. In other study by Bhat $\mathrm{BK}$ et $\mathrm{al}^{11}$ the incidence was found to be $0.74 \%$. Ramani CV et al, ${ }^{8}$ Chopra A et al, ${ }^{9}$ Khare AK et $\mathrm{al}^{10}{ }^{10}$ Ohana $\mathrm{N}$ et $\mathrm{al}^{13}$ have reported incidence of furunculosis as is $42.68 \%, 68.29 \%, 55.98 \%$ and $50 \%$ respectively. These findings are similar to the present study where incidence is $56 \%$.

We observed total $56 \%$ cases of furunculosis out of total 100 patients of bacterial folliculitis. Although 
patients of non-follicular pyoderma were excluded, total number of follicular and non-follicular bacterial infections in our study was 989. Hence a total proportion of furunculosis is $5.6 \%$. This coincides with the study of Chopra A et $\mathrm{al}^{9}$ where incidence was found to be $8.93 \%$. Mathew MS et $\mathrm{al}^{14}$ reported that $60 \%$ of the cases belonged to the rural area which coincides with the present study where $69 \%$ cases belonged to the rural areas. This is not surprising as most of the population our centre is from rural area and pyodermas are more common in rural population.

Chopra $\mathrm{A}$ et $\mathrm{al}^{9}$ and Khare $\mathrm{AK}$ et $\mathrm{al}^{10}$ have found higher incidence of bacterial skin infection in agriculturist and labourers which correlate with the present study (41\%). Mathew MS et $\mathrm{al}^{14}$ and Schachner $\mathrm{L}$ et $\mathrm{al}^{15}$ have reported an increased incidence of folliculitis in Lower socioeconomic status. Similar incidence found in our study. In the present study the male to female ratio 2.5:1 which was similar to the study conducted by Khare $\mathrm{AK}$ et $\mathrm{al}^{10}$ where the ratio was 1.8:1. In the present study folliculitis was frequently associated in the age group of 21-30 years. These findings coincide with the studies conducted by Khare AK et al. ${ }^{10}$ Anand LL et al $^{16}$ and also in present study it was found that most common symptom associated with superficial folliculitis is itch and that with furunculosis and carbuncles pain is chief symptom. Lymphadenitis was frequently associated with furunculosis although this may not be very common as skin infections are localized and are easily identified and treated in early stages of infection. Grouped lesions are observed in superficial folliculitis, chronic folliculitis of leg and sycosis barbae. This is because they spread peripherally to involve contagious hair follicles. However, single lesion was observed in carbuncle. Mathew MS et $\mathrm{al}^{14}$ observed increased frequency of superficial folliculitis and furunculosis on the legs which coincide with present study. However Venniyil PV et $\mathrm{al}^{6}$ found furunculosis to be the most common type of folliculitis in a recent Indian study. Chronic superficial folliculitis of legs exclusively presented on legs in present study. Legs are prone for local trauma and inoculation of the organisms leading to pyoderma including folliculitis. Sycosis barbae was found to be limited in beard area for obvious reasons as observed by Chopra A et al. ${ }^{9}$ Carbuncle presented most commonly on the nape of neck as observed by Chopra A et al and Bhat B.K.et al. ${ }^{9,11}$

Incidence of associated diseases like anaemia $25 \%$ cases, atopy in $31 \%$ cases, diabetes in $20 \%$ cases and HIV infection in $3 \%$ cases was found in present study of folliculitis. The incidence of anaemia in folliculitis is comparable to study of Ramani CK et al and Mathews MS et al. ${ }^{8,14}$ Anand LC et al ${ }^{16}$ observed that $25 \%$ cases of diabetes are associated with folliculitis. In present study Gram positive cocci were demonstrated in $73 \%$ cases citing importance of this easy-to-do procedure in dermatology practice. This should be done in early stages of infection and when patients are not treated with topical or systemic antibiotics. In the present study totally S. Aureus was isolated from $83 \%$ cases, S. Epidermidis from $8 \%$ and mixed from $7 \%$ cases. Similar findings were noted in studies of Khare AK et al. ${ }^{10}$

In the present study the organism isolated showed a high sensitivity to Netromycin, Ceftizoxime, Cefixime, and Gentamycin ranging from $80 \%$ to $95 \%$.There was $100 \%$ resistance to Penicillin $\mathrm{G}$ in the present study, which coincides with the observations of Ohana $\mathrm{N}$ et al. ${ }^{13}$ In the present study a high percentage $(43.96 \%)$ of resistance to Erythromycin was noted. This was similar to observations by Schachner $\mathrm{L}$ et al. ${ }^{15}$ Antibiotic sensitivity pattern is a useful guide for treating the disease with specific antibiotics. Use of ampicillin or penicillin is very common in general or dermatology practice. This study showed that majority of s. auerus isolated as pathogenic organism showed resistance to ampicillin. This also suggests use of cefixime or ciprofloxacin in these population to control the disease.

\section{Conclusion}

In the present study the proportion of folliculitis was $5.61 \%$ commonest in age group of 21 to 30 years mainly belonging to low socioeconomic status $(69 \%)$ mainly during Monsoon season (49\%). Aggravating factors are anaemia, atopy and diabetes responsible for chronicity of infection. Family history was present in $33 \%$ cases. Staphylococcus aureus was found in most $89 \%$ cases and epidermidis in $9 \%$ cases of superficial folliculitis. Beta hemolytic streptococcci was present as a mixed isolate in $7 \%$ cases. Staphylococcus aureus was found to be $100 \%$ penicillin resistant and $43.96 \%$ resistant to erythromycin in vitro. This study favours use of Cephalosporins as the first line of treatment in folliculitis on the basis of emerging resistance strains. This study demonstrates need of continuous monitoring of changing patterns of antibiotic sensitivity. It is evident that staphylococcus aureus is responsible for majority of cases of bacterial folliculitis and needs to be treated with appropriate antibiotics. S. epidermidis should no longer be considered as a harmless contaminant of the skin.

\section{References}

1. Ananthanarayana R, Paniker CKJ. Historical Introduction and Staphylococcus. In: Textbook of Microbiology. Second edition. Orient Longman: Bombay. 1981:1-7;175182.

2. Joseph DD. Bacterial Infections. In: Dermatologic Clinic. (Demis D. Joseph). First Edition. Vol. 3. Section 16. WB Saunders. London. 1980;16:2-16.

3. Konde S, Jairam LS, Peethambar P, Noojady SR, Kumar NC. Antibiotic over usage and resistance: A crosssectional survey among pediatric dentists. J Indian Soc Pedod Prev Dent._2016;34(2):145-51.

4. Miller RF. Epilating folliculitis of Glaborousskin. Report of a case, histopathologic and nasologic study. Arch Dermatol. 1961:83;777-84. 
5. Engman ME. Impetigo contagiosa, bullosa and its bacteriology J Cut Genit Urin. 1901:19;180-2.

6. Venniyil PV, Ganguly S, Kuruvila S, Devi S. A study of community-associated methicillin-resistant

Staphylococcus aureus in patients with pyoderma. Indian Dermatol Online J. 2016;7(3):159-63.

7. Valentine FC, Hall-Smith SP. Superficial staphylococcal infection. Lancet. 1952:2;351-54.

8. Ramani TV, Jayakar PA. Bacteriology of 100 cases of pyoderma. Ind J Dermatol Venereol Leprol. 1980;46:282284.

9. Chopra A, Purl R, Mittal RR. Correlation of isolates from pyoderma and carrier sites. Ind J Dermatol Venereol Leprol. 1995;61:273-75.

10. Khare AK. Clinical and bacteriological study of pyodermas. Ind J Dermatol Venereol Leprol. 1988:54;192-195.

11. Bhat BK, Nagesh CN. Drug resistance and penicillinase activity in skin isolated S. Aureus. Ind J Dermatol Venereol Leprol. 1990;56:371-374.
12. Tiwari VD, Ramaji C. Dermatitis cruris pustalosa et atrophicans. Ind J Dermatol Venereol Leprol. 1987;53:116-117.

13. Ohana N, Kennes J, Verner E, Raz R, Rozenman D, Zuckerman F. Skin isolated community acquired Staphylococcus aureus: In vitro resistance to methicillin and erythromycin. JAAD. 1989;21:544-46.

14. Mathew MS, Garg BR. A clinobacteriological study of primary pyoderma in children. Ind J Dermatol Venreol Leprol. 1992;58:183-187.

15. Schachner L, Gonzalez A. Staphylococcus aureus and erythromycin resistance in childhood pyoderma. Med J Aust. 1988;148(10):542-3.

16. Anand LC. Assessment of diabetic state in various skin disorders. Ind J Dermatol Venereol Leprosl. 1978:44;95103. 